\title{
Weighted Sums of Strongly Mixing Random Variables with an Application to Nonparametric Regression
}

\author{
Le Van Thanh* G. Yin ${ }^{\dagger}$
}

\begin{abstract}
This note establishes complete convergence for weighted sums of strongly mixing random variables. The result obtained is sharp. If the condition is relaxed slightly, the desired complete convergence does not hold, which is illustrated by two examples. An application of the main result to nonparametric regression is also considered.
\end{abstract}

Key words and phrases. Complete convergence, strongly mixing process, limit theorem, weighted sum, nonparametric regression.

AMS 2010 Subject Classifications. 60F15, 60G35.

*Department of Mathematics, Vinh University, Nghe An 42118, Vietnam. Email: levt@vinhuni.edu.vn. Research of this author was supported in part by NAFOSTED, grant no. 101.01.2012.13.

${ }^{\dagger}$ Department of Mathematics, Wayne State University, Detroit, MI 48202. Email: gyin@math.wayne.edu. Research of this author was supported in part by the National Science Foundation under DMS-1207667. 


\section{Introduction}

Mixing random processes, whose remote past and distant future are asymptotically independent, naturally arise in a wide range of applications. Their asymptotic properties are of crucial importance. Treating weighted sums of random variables, this work focuses on strongly mixing processes. Under appropriate conditions, we derive a strong limit theorem, which provides characterizations of the underlying processes from the angle of complete convergence. Such limit theorem for weighted sums has many important applications in stochastic control and statistics. Examples include state observers, least squares estimators, nonparametric regression function estimators, and jackknife estimates, among others; see for example, [14] and the references therein.

Let $(\Omega, \mathcal{F}, P)$ be a probability space and let $\mathcal{A}$ and $\mathcal{B}$ be two sub- $\sigma$-algebras of $\mathcal{F}$. Denote by $\mathcal{L}_{2}(\mathcal{A})$ the space of all square integrable and $\mathcal{A}$-measurable random variables. The strong mixing coefficient, $\varphi$-mixing coefficient, and maximal coefficient of correlation are defined by $\alpha(\mathcal{A}, \mathcal{B})=\sup \{|P(A B)-P(A) P(B)|: A \in \mathcal{A}, B \in \mathcal{B}\}, \varphi(\mathcal{A}, \mathcal{B})=\sup \{|P(B \mid A)-P(B)|:$ $A \in \mathcal{A}, B \in \mathcal{B}, P(A)>0\}$, and $\rho(\mathcal{A}, \mathcal{B})=\sup _{f \in \mathcal{L}_{2}(\mathcal{A}), g \in \mathcal{L}_{2}(\mathcal{B})}|\operatorname{corr}(f, g)|$, respectively. Let $X:=\left\{X_{k}, k \geq 1\right\}$ be a sequence of random variables. For $1 \leq J \leq L \leq \infty$, define the $\sigma$-field $\mathcal{F}_{J}^{L}=\sigma\left(X_{k}, J \leq k \leq L\right)$. Here and hereafter, for a subset $S$ of $\mathbb{N}=\{1,2, \ldots\}, \sigma(S)$ denotes the $\sigma$-field generated by $\left\{X_{n}, n \in S\right\}$. For $k \geq 1$, define $\alpha_{k}=\sup _{j \geq 1} \alpha\left(\mathcal{F}_{1}^{j}, \mathcal{F}_{j+k}^{\infty}\right)$, $\varphi_{k}=\sup _{j \geq 1} \varphi\left(\mathcal{F}_{1}^{j}, \mathcal{F}_{j+k}^{\infty}\right), \rho_{k}=\sup _{j \geq 1} \rho\left(\mathcal{F}_{1}^{j}, \mathcal{F}_{j+k}^{\infty}\right)$, and $\rho_{k}^{*}=\sup \rho(\sigma(S), \sigma(T))$, where the sup is taken over all pairs of nonempty finite sets $S, T$ of $\mathbb{N} \operatorname{such}$ that $\operatorname{dist}(S, T) \geq k$. The sequence $\left\{X_{i}, i \geq 1\right\}$ is said to be strongly mixing (respectively, $\varphi$-mixing, $\rho$-mixing, $\rho^{*}$ mixing) if $\alpha_{k} \rightarrow 0$ (respectively, $\varphi_{k} \rightarrow 0, \rho_{k} \rightarrow 0, \rho_{k}^{*} \rightarrow 0$ ) as $k \rightarrow \infty$.

In many applications, the mixing rates are difficult to estimate; see for example, [14]. Therefore it is interesting to replace the mixing rates by sufficient conditions imposed to other dependence coefficients that might be easier to verify in certain cases. The weaker mixing condition is based on the following coefficient. For $k \geq 1$, let $r_{k}^{*}=\sup |\operatorname{corr}(V ; W)|$, where the supreme is taken over all nonempty finite subsets $S, T$ of $\{1,2, \ldots\}$ such that $\operatorname{dist}(S, T) \geq k$ and all the linear combinations $V=\sum_{i \in S} a_{i} X_{i}$ and $W=\sum_{i \in T} b_{i} X_{i}$. For stationary Gaussian sequences, the coefficients $\rho_{k}^{*}$ and $r_{k}^{*}$ are identical [6]. Throughout the paper, we use the symbols $C, K$ to denote a generic positive constants, whose values may be different for different appearances. For $x>0$, let $\lfloor x\rfloor$ denote the greatest integer less than 
or equal to $x$ and let $\log x$ denote $\log _{2} x$.

In this paper, by considering weighted sums of strongly mixing sequences, we provide sufficient conditions for complete convergence. Our Theorem 2.2 extends the main result of Shao [11] to the case of weighted sums. Two illustrative examples are provided to show that the conditions on the weights and required moments cannot be weakened. For complete convergence for other type of mixing dependence, see Peligrad and Gut [7], Sung [13], Wu et al. [15] for $\rho^{*}$-mixing processes, and Shen et al. [12] for $\varphi$-mixing processes. The scaling factor $n^{1 / r}(\log n)^{\beta}$ was used in [13] and [15], whereas we use $n^{1 / r}$ instead. Also our proofs are different from theirs; see also the comments after Lemma 2.1.

The rest of the paper is arranged as follows. Section 2 presents complete convergence for weighted sums. Section 3 proceeds with an application to demonstrate the main result.

\section{Complete Convergence for Weighted Sums}

The concept of complete convergence was introduced in [5]. A sequence of random variables $\left\{X_{n}, n \geq 1\right\}$ is said to converge completely to 0 if

$$
\sum_{n=1}^{\infty} P\left(\left|X_{n}\right|>\varepsilon\right)<\infty \text { for all } \varepsilon>0 .
$$

Based on this definition, Hsu and Robbins [5] and Erdös [3] derived a necessary and sufficient condition for a sequence of independent identically distributed (i.i.d.) random variables $\left\{X_{n}, n \geq 1\right\}$. Their assertion reads: $E X_{1}=0$ and $E\left|X_{1}\right|^{2}<\infty$ if and only if

$$
\sum_{n=1}^{\infty} P\left(\left|\sum_{i=1}^{n} X_{i}\right|>\varepsilon n\right)<\infty \text { for all } \varepsilon>0 .
$$

Their theorem was extended by Baum and Katz [1] as follows: Let $\eta>1 / 2, p \geq 1$, and $\left\{X_{n}, n \geq 1\right\}$ be a sequence of i.i.d. random variables. Then $E X_{1}=0$ and $E\left|X_{1}\right|^{p}<\infty$ if and only if

$$
\sum_{n=1}^{\infty} n^{\eta p-2} P\left(\left|\sum_{i=1}^{n} X_{i}\right|>\varepsilon n^{\eta}\right)<\infty \text { for all } \varepsilon>0 .
$$

Motivated by applications in sequential analysis and renewal theory, many researchers extended the complete convergence to weakly dependent random variables, including $\rho$ mixing (Shao [10]) and strongly mixing (Berbee [2]). Shao [11] extended the result of Berbee [2] from bounded random variables to unbounded random variables. In this section, we 
establish the complete convergence for weighted sums of strongly mixing random variables. The following lemma is a maximal type inequality for strongly mixing random variables; see [11] for a proof.

Lemma 2.1. Let $\left\{X_{i}, 1 \leq i \leq n\right\}$ be a sequence of zero-mean random variables. Then

$$
P\left(\max _{j \leq n}\left|\sum_{i=1}^{j} X_{i}\right| \geq x\right) \leq \frac{4}{x} \sum_{i=1}^{n} E\left|X_{i}\right| I\left(\left|X_{i}\right|>c\right)+\frac{4}{x^{a}}+\frac{32^{3} n c \alpha_{k}}{x}
$$

for any $a \geq 1, x \geq 1, c>0$ and integer $k$ satisfying the following conditions

$$
1 \leq k \leq \frac{x}{64 a c \log (x \vee 2)}
$$

and

$$
\left(\sum_{i=1}^{n} E\left(\left|X_{i}\right|^{s} I\left(\left|X_{i}\right| \leq c\right)\right)^{2 / s}\right)\left(\sum_{i=0}^{k} \alpha_{i}^{1-2 / s}\right) \leq \frac{x^{2}}{32^{3} a \log (x \vee 2)} \text { for some } s \geq 2 \text {. }
$$

A main tool of the proof of complete convergence theorems for weighted sums is the Rosenthal-type inequality (see, e.g., Rosenthal [8]) for maximum partial sums as pointed out by Sung [13]. Zhou and Lin [17] used the Rosenthal-type inequality in a slightly weaker form [17, Lemmas 2.1 and 2.2] to derive results on complete convergence for weighted sums (see [17, Theorems 1.3 and 1.4]) that are similar to Theorem 2.2 under a faster decay of strongly mixing rate; see Remark 2.4 for details. The maximal inequality in Lemma 2.1 is very different and the proof of the limit theorems for strongly mixing presented in this paper is more complicate. While the proof of our main result relies on Lemma 2.1, the weighted sums presented here is of interest in its own right; see Example 2.6, Example 2.7 and Proposition 3.1. The following theorem reduces to the main result of Shao [11] when $a_{n i} \equiv 1$ a.s. We note that the mixing rate in (2.7) below is exactly the same as the mixing rate in [11].

Theorem 2.2. Let $\eta>1 / 2, q>p \geq 1 / \eta \geq 1$, and $\left\{X_{n}, n \geq 1\right\}$ be a strongly mixing sequence of mean-zero random variables such that $\sup _{n \geq 1} E\left|X_{n}\right|^{q} \leq 1$. Let $\left\{a_{n i}, i \geq 1, n \geq 1\right\}$, be an array of constants. Assume that

$$
\alpha_{k}=O\left(\frac{1}{k^{q(p-1) /(q-p)} \log ^{t} k}\right) \text { for some } t>\frac{p q}{q-p} .
$$

If

$$
\sum_{i=1}^{n}\left|a_{n i}\right|^{q}=O(n)
$$


then

$$
\sum_{n=1}^{\infty} n^{\eta p-2} P\left\{\max _{j \leq n}\left|\sum_{i=1}^{j} a_{n i} X_{i}\right| \geq \varepsilon n^{\eta}\right\}<\infty \text { for all } \varepsilon>0
$$

Proof. Set

$$
M=\sup _{n \geq 1} \frac{\sum_{i=1}^{n}\left|a_{n i}\right|^{q}}{n}+1 .
$$

It follows from $(2.8)$ that $M<\infty$. Setting $u=q(p-1) /(q-p)$, we will prove that there exists a sufficiently large constant $K$ being independent of $n$ such that

$$
P\left\{\max _{j \leq n}\left|\sum_{i=1}^{j} a_{n i} X_{i}\right| \geq x\right\} \leq K n x^{-q(u+1) /(q+u)} \log ^{(q-1)(u-t) /(q+u)} x
$$

for all large $n$ and for all $x \geq K n^{1 / 2} \log ^{1+t / 2} n$. If $K n x^{-q(u+1) /(q+u)} \log ^{(q-1)(u-t) /(q+u)} x>1$, then (2.11) is trivial. Therefore we can assume

$$
K n x^{-q(u+1) /(q+u)} \log ^{(q-1)(u-t) /(q+u)} x \leq 1 .
$$

Set

$$
c=2 x^{u /(q+u)} \log ^{(t-u) /(q+u)} x, a=u+2, k=\left\lfloor\frac{x}{64 a c \log x}\right\rfloor .
$$

Case 1: $1<q \leq 2$. In this case, we have

$$
\begin{aligned}
(k & +1)\left(\sum_{i=1}^{n} E\left(a_{n i} X_{i} I\left(\left|a_{n i} X_{i}\right| \leq c\right)\right)^{2}\right) \\
& \leq 2 k\left(\sum_{i=1}^{n}\left|a_{n i}\right|^{q} E\left|X_{i}\right|^{q}\right) c^{2-q} \\
& \leq M \frac{n x c^{1-q}}{32 a \log x} \\
& =\frac{x^{2}}{32^{3} a \log x} 32^{2} M 2^{1-q} n x^{-q(u+1) /(q+u)} \log ^{(q-1)(u-t) /(q+u)} x \\
& \leq \frac{x^{2}}{32^{3} a \log x}(\text { by }(2.12)) .
\end{aligned}
$$

By (2.13) and (2.14), we can apply Lemma 2.1 with $s=2$ to obtain

$$
P\left\{\max _{j \leq n}\left|\sum_{i=1}^{j} a_{n i} X_{i}\right| \geq x\right\} \leq C\left(\frac{1}{x} \sum_{i=1}^{n} E\left|a_{n i} X_{i}\right| I\left(\left|a_{n i} X_{i}\right|>c\right)+\frac{1}{x^{a}}+\frac{n c \alpha_{k}}{x}\right) .
$$


Since $q>1$ and $\sup _{n \geq 1} E\left|X_{n}\right|^{q} \leq 1$,

$$
\begin{aligned}
& \frac{1}{x} \sum_{i=1}^{n} E\left|a_{n i} X_{i}\right| I\left(\left|a_{n i} X_{i}\right|>c\right) \\
& \quad \leq \frac{c^{1-q}}{x} \sum_{i=1}^{n}\left|a_{n i}\right|^{q} \\
& \quad \leq \frac{M n c^{1-q}}{x}(\text { by }(2.10)) \\
& \quad \leq M n x^{-q(u+1) /(q+u)} \log ^{(q-1)(u-t) /(q+u)} x(\text { by }(2.13)) .
\end{aligned}
$$

By an elementary computation using (2.7), we also have

$$
\frac{1}{x^{a}}+\frac{n c \alpha_{k}}{x} \leq C n x^{-q(u+1) /(q+u)} \log ^{(q-1)(u-t) /(q+u)} x .
$$

Conclusion (2.11) follows from (2.15)-(2.17).

Case 2: $q>2$. In this case, we have

$$
\begin{aligned}
& \left(\sum_{i=1}^{n}\left(E\left|a_{n i} X_{i} I\left(\left|a_{n i} X_{i}\right| \leq c\right)\right|^{q}\right)^{2 / q}\right) \sum_{i=0}^{k} \alpha_{i}^{1-2 / q} \\
& \leq\left(\sum_{i=1}^{n}\left|a_{n i}\right|^{2}\right) \sum_{i=0}^{k} \alpha_{i}^{1-2 / q} \\
& \leq\left(\sum_{i=1,\left|a_{n i}\right| \leq 1}^{n}\left|a_{n i}\right|^{2}\right) \sum_{i=0}^{k} \alpha_{i}^{1-2 / q}+\left(\sum_{i=1,\left|a_{n i}\right|>1}^{n}\left|a_{n i}\right|^{q}\right) \sum_{i=0}^{k} \alpha_{i}^{1-2 / q} \\
& \leq(1+M) n \sum_{i=0}^{k} \alpha_{i}^{1-2 / q}(\text { by }(2.10)) \\
& \leq(1+M) n\left(1+C \sum_{i=1}^{k} i^{-u(1-2 / q)} \log ^{-t(1-2 / q)} i\right) \\
& \leq C n\left(\log { }^{1+t(1-2 / q)} k+k^{1-u(1-2 / q)} \log ^{-t(1-2 / q)} k\right) \\
& \leq \frac{x^{2}}{32^{3} a \log x} \text { (by the last four lines of (20) in Shao [11, page 284]). }
\end{aligned}
$$

By (2.13) and (2.18), we can again apply Lemma 2.1 with $s=q$ to obtain (2.15). Similar to the case $1<q \leq 2,(2.11)$ still holds.

Now we prove (2.9). Let $\varepsilon>0$. Since $\eta>1 / 2$, given a constant $C, \varepsilon n^{\eta} \geq C n^{1 / 2} \log ^{1+t / 2} n$ 
for all large $n$. It follows from (2.11) that

$$
\begin{aligned}
& \sum_{n=1}^{\infty} n^{\eta p-2} P\left\{\max _{j \leq n}\left|\sum_{i=1}^{j} a_{n i} X_{i}\right| \geq \varepsilon n^{\eta}\right\} \\
& \leq K \sum_{n=1}^{\infty} n^{\eta p-2} n^{1-\eta q(u+1) /(q+u)} \log ^{(q-1)(u-t) /(q+u)} n \\
& =K \sum_{n=1}^{\infty} n^{-1} \log ^{-1-\beta} n
\end{aligned}
$$

where $\beta=-\frac{(q-1)(u-t)}{q+u}-1=\frac{t(q-p)}{q}-p>0$. Therefore, (2.9) follows.

Remark 2.3. It is clear that $\sup _{n \geq 1} E\left|X_{n}\right|^{q} \leq 1$ can be replaced by $\sup _{n \geq 1} E\left|X_{n}\right|^{q} \leq C<\infty$.

Remark 2.4. Recently, Zhou and Lin [17] also examined complete convergence of strongly mixing processes. Let us briefly compare their results with ours.

(i) Theorem $1.3[17]$ required that

$$
\alpha_{k}=O\left(\frac{1}{k^{\theta}}\right) \text { for some } \theta>\frac{q(q+\delta)}{2 \delta}, \delta>0, q>2 .
$$

In Theorem 2.2 of this paper, by setting $\delta=(q-p) / 2>0,(2.7)$ becomes

$$
\alpha_{k}=O\left(\frac{1}{k^{q(q-2 \delta-1) /(2 \delta)} \log ^{t} k}\right) \text { for some } t>\frac{q(q-2 \delta)}{2 \delta} .
$$

The strong mixing rate in (2.19) decays much faster than that in (2.20). Theorem 1.3 in [17] required $\left\{X_{n}, n \geq 1\right\}$ be identically distributed random variables with $E\left|X_{1}\right|^{p+\delta}<\infty$. In Theorem 2.2, we do not require $\left\{X_{n}, n \geq 1\right\}$ be identically distributed, but we need slightly stronger moment condition $\sup _{n \geq 1} E\left|X_{n}\right|^{p+2 \delta}<\infty$.

(ii) In our Theorem 2.2, by setting $\delta=q-p>0$, (2.7) becomes

$$
\alpha_{k}=O\left(\frac{1}{k^{q(q-\delta-1) / \delta} \log ^{t} k}\right) \text { for some } t>\frac{q(q-\delta)}{\delta} .
$$

If $q \leq 2+3 \delta$ that is equivalent to

$$
2 q \geq 3 p-2,
$$

then by elementary computation, we have $q(q-\delta-1) / \delta \leq q(q+\delta) /(2 \delta)$. That is, our strong mixing rate is better than that required in [17, Theorem 1.3]. Our condition on the weights is $\sum_{i=1}^{n}\left|a_{n i}\right|^{q}=\sum_{i=1}^{n}\left|a_{n i}\right|^{p+\delta}=O(n)$, weaker than $\sum_{i=1}^{n}\left|a_{n i}\right|^{p+\delta}=O\left(n^{t}\right)$ for some $0<t<1$, used in [17, Theorem 1.3]. Note that the condition used in [17, Theorem 1.3] is not satisfied even when $a_{n i} \equiv 1$. 
(iii) Since $q>p,(2.22)$ is satisfied when $p \leq 2$. By letting $\delta=q-p$, all assumptions of Theorem 2.2 are weaker than that of [17, Theorem 1.1].

(iv) In [17, Theorems 1.2 and 1.4], the authors required that the strong mixing rate $\alpha_{k}$ decays faster than

$$
\frac{C}{k^{\theta}} \text { for some } \theta>\frac{(q-1)(q+\delta)}{\delta}, \delta>0, q>2 .
$$

In Theorem 2.2, if we set $\delta=q-p>0$ as in (ii), then strongly mixing rate in (2.21) is much slower than that in (2.23). Therefore, Theorem 2.2 extends Theorems 1.2 and 1.4 in [17] in three directions: (a) our strong mixing rate decays much slower, (b) $X_{n}$ need not be identically distributed, and (c) our condition on the weights is weaker.

The following corollary provides sufficient conditions for almost sure convergence of weighted sums.

Corollary 2.5. Let $1 \leq r<2, q>2 r$ and $\left\{X_{n}, n \geq 1\right\}$ be a strongly mixing sequence of mean-zero random variables such that $\sup _{n \geq 1} E\left|X_{n}\right|^{q}<\infty$. Let $\left\{a_{n i}, i \geq 1, n \geq 1\right\}$ be an array of constants. Assume that

$$
\alpha_{k}=O\left(\frac{1}{k^{q(2 r-1) /(q-2 r)} \log ^{t} k}\right) \text { for some } t>\frac{2 q r}{q-2 r} .
$$

If

$$
\sum_{i=1}^{n}\left|a_{n i}\right|^{q}=O(n)
$$

then

$$
\sum_{n=1}^{\infty} P\left\{\max _{j \leq n}\left|\sum_{i=1}^{j} a_{n i} X_{i}\right| \geq \varepsilon n^{1 / r}\right\}<\infty \text { for all } \varepsilon>0
$$

and therefore

$$
\lim _{n \rightarrow \infty} \frac{\sum_{i=1}^{n} a_{n i} X_{i}}{n^{1 / r}}=0 \text { a.s. }
$$

Proof. Let $p=2 r$ and $\eta=2 / p$. The corollary follows immediately from Theorem 2.2 .

How sharp is our result? In Corollary 2.5, if we take $r=1$, then we require $\sup _{n \geq 1} E\left|X_{n}\right|^{q}<$ $\infty$ for some $q>2$. Comparing with (2.2) and (2.3), it may seem unnecessary to have moments of order strictly large than 2 to get the rates of convergence in the strong law of large numbers. An interesting question is: Can we replace the condition $\sup _{n \geq 1} E\left|X_{n}\right|^{q}<\infty$ in Corollary 2.5 with a slightly weaker condition

$$
\sup _{n \geq 1} E\left(\left|X_{n}\right|^{2 r} \log \left(\left|X_{n}\right| \vee 2\right)\right)<\infty ?
$$


The following example shows that Corollary 2.5 can fail if the condition $\sup _{n \geq 1} E\left|X_{n}\right|^{q}<\infty$ is replaced by (2.27), and (2.24) is replaced by

$$
\sum_{i=1}^{n} E\left|a_{n i}\right|^{2 r}=O(n)
$$

Example 2.6. Let $r=1$ and consider a sequence $\left\{X_{n}, n \geq 1\right\}$ of independent 0 mean random variables such that for all $n \geq 1$

$P\left\{X_{n}=0\right\}=1-1 /(n \log (n \vee 2))$ and $P\left\{X_{n}=-n^{1 / 2}\right\}=P\left\{X_{n}=n^{1 / 2}\right\}=1 /(2 n \log (n \vee 2))$

Then $\sup _{n \geq 1} E\left|X_{n}\right|^{q}=\infty$ for all $q>2 r$ but

$$
\sup _{n \geq 1} E\left(\left|X_{n}\right|^{2 r} \log \left(\left|X_{n}\right| \vee 2\right)\right)=\sup _{n \geq 1} E\left(\left|X_{n}\right|^{2} \log \left(\left|X_{n}\right| \vee 2\right)\right)=1 / 2<\infty
$$

i.e., (2.27) holds. For $n \geq 1$, let $a_{n i}=0$ for all $1 \leq i<n$ and $a_{n n}=n^{1 / 2}$. Then (2.28) holds since for all $n \geq 1 \sum_{i=1}^{n} E\left|a_{n i}\right|^{2 r}=\sum_{i=1}^{n} E\left|a_{n i}\right|^{2}=n$. However, for all $0<\varepsilon \leq 1$,

$$
\sum_{n=2}^{\infty} P\left\{\max _{j \leq n}\left|\sum_{i=1}^{j} a_{n i} X_{i}\right| \geq \varepsilon n^{1 / r}\right\}=\sum_{n=2}^{\infty} P\left\{n^{1 / 2}\left|X_{n}\right| \geq \varepsilon n\right\}=\sum_{n=2}^{\infty} \frac{1}{n \log n}=\infty
$$

i.e., (2.25) fails. By applying the Borel-Cantelli lemma, we conclude that (2.26) fails as well.

In Example 2.6, for all $q>2, \sup _{n \geq 1} E\left|X_{n}\right|^{q}=\infty$ and $\sum_{i=1}^{n}\left|a_{n i}\right|^{q}=n^{q / 2}>n$ for all $n \geq 2$. The following example, which is a modification of Example 2.6, shows that even when $\sup _{n \geq 1} E\left|X_{n}\right|^{q}<\infty$, the condition (2.8) in Theorem 2.2 cannot be replaced by a weaker condition

$$
\sum_{i=1}^{n}\left|a_{n i}\right|^{q}=O\left(n^{1+\delta}\right) \text { for some } \delta>0
$$

Example 2.7. Let $p=2 \eta=2, \delta>0, q=2+\delta$ and consider a sequence $\left\{X_{n}, n \geq 1\right\}$ of independent mean 0 random variables such that for all $n \geq 1$

$$
P\left\{X_{n}=0\right\}=1-1 / n \text { and } P\left\{X_{n}=-n^{1 /(2+\delta)}\right\}=P\left\{X_{n}=n^{1 /(2+\delta)}\right\}=1 /(2 n) .
$$

Then $\sup _{n \geq 1} E\left|X_{n}\right|^{q}=1<\infty$. For $n \geq 1$, let $a_{n i}=0$ for all $1 \leq i<n$ and $a_{n n}=n^{(1+\delta) /(2+\delta)}$. Then for all $n \geq 1, \sum_{i=1}^{n} E\left|a_{n i}\right|^{q}=n^{1+\delta}$, i.e., (2.29) holds but (2.8) fails. However, for all $0<\varepsilon \leq 1$,

$$
\sum_{n=1}^{\infty} n^{\eta p-2} P\left\{\max _{j \leq n}\left|\sum_{i=1}^{j} a_{n i} X_{i}\right| \geq \varepsilon n^{\eta}\right\}=\sum_{n=1}^{\infty} P\left\{n^{1 / 2}\left|X_{n}\right| \geq \varepsilon n\right\}=\sum_{n=1}^{\infty} \frac{1}{n}=\infty
$$




\section{An Application to Nonparametric Multiple Regres- sion}

This section is devoted to a statistical application of Corollary 2.5. We consider the nonparametric multiple regressions as follows.

Let $p$ be a positive integer and $A$ be a compact set in $\mathbb{R}^{p}$. Consider the following observations $Y_{n i}=g\left(x_{n i}\right)+X_{n i}, 1 \leq i \leq n$, where $g$ is an unknown real-valued regression function that is bounded on $A, x_{n i}$ are known fixed design points from $A,\left\{X_{n i}\right\}$ is a sequence random variables representing the random errors. As in $[9,16]$, we assume that for each $n$, there is $\left\{X_{i}, i \geq 1\right\}$, a sequence of strongly mixing random variables such that

$$
\left\{X_{n i}, 1 \leq i \leq n\right\} \text { has the same joint distribution as }\left\{X_{i}, 1 \leq i \leq n\right\} .
$$

We consider the following weighted regression estimator of $g: g_{n}(x)=\sum_{i=1}^{n} W_{n i}(x) Y_{n i}$, $x \in A$, where the weight functions $W_{n i}(x)$ depend on the fixed design points $x_{n i}, 1 \leq i \leq n$, and on the number of observations $n$. The following theorem gives sufficient conditions for complete consistency of the estimate $g_{n}(x)$.

Proposition 3.1. Let the weight functions satisfy that as $n \rightarrow \infty$

$$
\begin{gathered}
\sum_{i=1}^{n} W_{n i}(x) \rightarrow 1, \\
\sum_{i=1}^{n}\left|W_{n i}(x)\right|=O(1),
\end{gathered}
$$

and

$$
\sum_{i=1}^{n}\left|W_{n i}(x)\right| I\left(\left\|x_{n i}-x\right\|>a\right) \rightarrow 0 \text { for all } a>0 .
$$

Let $1 \leq r<2, q>2 r$ and $\left\{X_{i}, i \geq 1\right\}$ be a strongly mixing sequence of mean-zero random variables such that

$$
\alpha_{k}=O\left(\frac{1}{k^{q(2 r-1) /(q-2 r)} \log ^{t} k}\right) \text { for some } t>\frac{2 q r}{q-2 r} .
$$

If $\sup _{i \geq 1} E\left|X_{i}\right|^{q}<\infty$ and

$$
\sup _{1 \leq i \leq n}\left|W_{n i}(x)\right|=O\left(n^{\frac{r-q}{r(q-1)}}\right),
$$

then for any continuity point $x \in A$ of the function $g$,

$$
g_{n}(x) \rightarrow g(x) \text { completely }
$$


Proof. Note that

$$
\begin{aligned}
\left|E g_{n}(x)-g(x)\right| \leq & \sum_{i=1}^{n}\left|W_{n i}(x)\right|\left|g\left(x_{n i}\right)-g(x)\right|\left(I\left(\left\|x_{n i}-x\right\| \leq a\right)+I\left(\left\|x_{n i}-x\right\|>a\right)\right) \\
& +|g(x)|\left|\sum_{i=1}^{n} W_{n i}(x)-1\right| .
\end{aligned}
$$

Then by (3.1), (3.2), (3.3) and the boundedness and continuity at $x$ of $g$,

$$
\left|E g_{n}(x)-g(x)\right| \rightarrow 0 \text { as } n \rightarrow \infty
$$

Since

$$
\begin{aligned}
\left|g_{n}(x)-g(x)\right| & \leq\left|g_{n}(x)-E g_{n}(x)\right|+\left|E g_{n}(x)-g(x)\right| \\
& =\left|\sum_{i=1}^{n} W_{n i}(x) X_{n i}\right|+\left|E g_{n}(x)-g(x)\right|,
\end{aligned}
$$

it suffices to prove that

$$
\left|\sum_{i=1}^{n} W_{n i}(x) X_{n i}\right| \rightarrow 0 \text { completely as } n \rightarrow \infty .
$$

Now let $a_{n i}=n^{1 / r} W_{n i}(x), 1 \leq i \leq n$. By (3.2) and (3.4),

$$
\begin{aligned}
\sum_{i=1}^{n}\left|a_{n i}\right|^{q} & =n^{q / r} \sum_{i=1}^{n}\left|W_{n i}(x)\right|^{q} \\
& \leq n^{q / r}\left(\sum_{i=1}^{n}\left|W_{n i}(x)\right|\right) \sup _{1 \leq i \leq n}\left|W_{n i}(x)\right|^{q-1}=O(n) \text { as } n \rightarrow \infty .
\end{aligned}
$$

Thus, Corollary 2.5 leads to

$$
\left|\frac{1}{n^{1 / r}} \sum_{i=1}^{n} a_{n i} X_{i}\right|=\left|\sum_{i=1}^{n} W_{n i}(x) X_{i}\right| \rightarrow 0 \text { completely as } n \rightarrow \infty .
$$

Since for each $n \geq 1,\left\{X_{n i}, 1 \leq i \leq n\right\}$ has the same joint distribution as $\left\{X_{i}, 1 \leq i \leq n\right\}$, (3.10) yields (3.9).

Remark 3.2. Proposition 3.1 should be compared with Theorem 5 in Georgiev [4], in which the random errors are independent and stochastically dominated.

\section{Acknowledgements}

We are very grateful to the editors and the referees for their constructive comments and detailed suggestions that lead to much improvement of the paper. 


\section{References}

[1] L.E. Baum and M. Katz, Convergence rates in the law of large numbers. Trans. Amer. Math. Soc. 120 (1965), 108-123.

[2] H. C. P. Berbee, Convergence rates in the strong law for bounded mixing sequences. Probab. Theory Rel. Fields, 74 (1987), 255-270.

[3] P. Erdös, On a theorem of Hsu and Robbins. Ann. Math. Statist. 20 (1949), 286-291.

[4] A. Georgiev, Consistent nonparametric multiple regression: the fixed design case. J. Multivariate Anal. 25 (1988), no. 1, 100-110.

[5] P.L. Hsu, H. Robbins, Complete convergence and the law of large numbers. Proc. Nat. Acad. Sci. U. S. A., 33 (1947), 25-31.

[6] A.N. Kolmogorov and Y.A. Rozanov, On strong mixing conditions for stationary Gaussian processes. Theory Probab. Appl. 5 (1960), 204-208.

[7] M. Peligrad, A. Gut, Almost-sure results for a class of dependent random variables. J. Theoret. Probab. 12 (1999), 87-104.

[8] H. P. Rosenthal, On the subspaces of $L^{p}(p>2)$ spanned by sequences of independent random variables. Israel J. Math. 8 (1970), 273-303.

[9] G. G. Roussas, T. L. Tran, and D. A. Ioannides, Fixed design regression for time series: asymptotic normality, J. Multivariate Anal. 40 (1992), no. 2, 262-291.

[10] Q. M. Shao, Maximal inequalities for partial sums of $\rho$-mixing sequences. Ann. Probab. 23 (1995), no. 2, 948-965.

[11] Q. M. Shao, Complete convergence for $\alpha$-mixing sequences. Statist. Probab. Lett. 16 (1993), no. 4, 279-287.

[12] A. Shen, X. Wang, X. Li, and X. Wang, On the rate of complete convergence for weighted sums of arrays of rowwise -mixing random variables. Comm. Statist. Theory Methods 43 (2014), no. $13,2714-2725$.

[13] S. H. Sung, On the strong convergence for weighted sums of $\rho^{*}$-mixing random variables. Statist. Papers 54 (2013), no. 3, 773-781.

[14] L. V. Thanh, G. Yin, and L.Y. Wang, State observers with random sampling times and convergence analysis of double-indexed and randomly-weighted sums of mixing processes. SIAM J. Control Optim. 49 (2011), no. 1, 106-124.

[15] Y. Wu, S. H. Sung, and A. Volodin, A note on the rates of convergence for weighted sums of $\rho^{*}$-mixing random variables. Lith. Math. J. 54 (2014), no. 2, 220-228.

[16] W. Z. Yang, X. J. Wang, X. H. Wang, and S. H. Hu, The consistency for estimator of nonparametric regression model based on NOD errors. J. Inequal. Appl. 2012, 2012:140, 13 pp.

[17] X. C. Zhou and J. G. Lin, On complete convergence for strong mixing sequences, Stochastics, (2013), no.2, 262-271. 